\title{
Lineamientos para una política pública de la pesca artesanal. El caso del puerto de Matarani
}

Guidelines for a public policy of artisanal fishing. The case of the port of Matarani

Diretrizes para uma política pública de pesca artesanal. O caso do porto de Matarani

Artículo resultado parcial del proyecto de investigación TP-029-2018 titulado: "Estudio del impacto de la pesca industrial y las políticas pesqueras en la condición sociocultural de los pescadores artesanales del puerto de Matarani, distrito de Islay, provincia de Islay de la región Arequipa", financiado por la Universidad Nacional de San Agustín de Arequipa.

\section{ARTÍCULO ORIGINAL}

\section{Escalante Diaz, Jean Pierre \\ https://orcid.org/0000-0003-2071-1742 jescalanted@unsa.edu.pe}

Bachiller en Antropología por la Universidad Nacional de San Agustín de Arequipa, Perú.

\section{Mamani Daza, Lolo Juan \\ Imamanid@unsa.edu.pe \\ https://orcid.org/0000-0002-7922-1716}

Doctor en Ciencias Sociales. Magíster en Ciencias Sociales con mención en Estrategias de Desarrollo y Políticas Sociales

Recibido 05 de Febrero 2021 | Arbitrado y aceptado 19 de Abril 2021 | Publicado en 28 Julio 2021

\section{RESUMEN}

Las sociedades costeras asentadas en el litoral arequipeño tienen una larga tradición como pescadores artesanales. Sin embargo las relaciones económicas, sociales, políticas y el imaginario simbólico del pescador han ido cambiando en las últimas décadas como respuesta al impacto de la modernidad y de las políticas públicas vigentes. En ese sentido, el presente artículo tiene como objetivo proponer algunos lineamientos para el desarrollo de una política pública para la pesca artesanal en el Puerto de Matarani, que permita comprender los modos de inclusión de una sociedad pesquera artesanal en un nuevo entorno regional bajo el impacto de la modernidad. Para este fin, la presente investigación es de tipo correlacional, aplicando una metodología empírico-analítica bajo un modelo hipotético-deductivo. Para la recolección de datos, se utilizó la técnica de la encuesta y entrevista, mediante un cuestionario con escala tipo Likert y de preguntas abiertas, aplicado a una muestra de 306 pescadores artesanales del Puerto de Matarani. Los resultados expresan que la construcción de la política pública es ineludible si se quiere hacer sostenible la pesca artesanal. Concluyéndose, que es necesario hacer énfasis en la gestión de la información, el fortalecimiento institucional, el ordenamiento pesquero, la investigación y el cambio climático.

Palabras clave: Pesca Artesanal, Puerto de Matarani, modernidad, política pública, pescadores artesanales.

\section{ABSTRACT}

The coastal societies settled on the Arequipa coast have a long tradition as artisanal fishermen. However, economic, social and political relations and the symbolic imagination of the fisherman have been changing in recent decades in response to the impact of modernity and current public policies. In this sense, this article aims to propose some guidelines for the development of a public policy for artisanal fishing in the Port of Matarani, which allows understanding the modes of inclusion of an artisanal fishing society in a new regional environment under the impact of modernity. For this purpose, the present investigation is of a correlational type, applying an empirical-analytical methodology under a hypothetical-deductive model. For data collection, the survey and interview technique was used, through a questionnaire with a Likert-type scale and open questions, applied to a sample of 306 artisanal fishermen from the Port of Matarani. The results express that the construction of public policy is unavoidable if artisanal fishing is to be made sustainable.

Concluding, that it is necessary to emphasize information management, institutional strengthening, fisheries management, research and climate change.

Keywords: Artisanal Fishing, Port of Matarani, modernity, public policy, artisanal fishermen.

\section{RESUMO}

As sociedades costeiras instaladas na costa de Arequipa têm uma longa tradição como pescadores artesanais. No entanto, as relações econômicas, sociais e políticas e o imaginário simbólico do pescador vêm mudando nas últimas décadas em resposta aos impactos da modernidade e das políticas públicas vigentes. Nesse sentido, este artigo tem como objetivo propor algumas diretrizes para o desenvolvimento de uma política pública para a pesca artesanal no Porto de Matarani, que permita compreender os modos de inserção de uma sociedade de pesca artesanal em um novo ambiente regional sob o impacto da modernidade. Para tanto, a presente investigação é do tipo correlacional, aplicando uma metodologia empíricoanalítica sob um modelo hipotéticodedutivo. Para a coleta de dados, utilizouse a técnica de survey e entrevista, por meio de um questionário com escala do tipo Likert e questões abertas, aplicado a uma amostra de 306 pescadores artesanais do Porto de Matarani. Os resultados expressam que a construção de políticas públicas é imprescindível para que a pesca artesanal seja sustentável. Concluindo, é necessário enfatizar a gestão da informação, o fortalecimento institucional, a gestão pesqueira, a pesquisa $\mathrm{e}$ as mudanças climáticas.

Palavras-chave: Pesca Artesanal, Porto de Matarani, modernidade, políticas públicas, pescadores artesanais. 


\section{Introducción}

El Perú es reconocido en el mundo como una potencia en pesca debido a sus altos índices de productividad, así como por la abundancia y diversidad de los recursos hidrobiológicos que brinda su ecosistema marino. Según la Organización de las Naciones Unidas para la Alimentación y la Agricultura (2019) es el segundo país en el mundo en materia de capturas marinas, habiendo registrado 7.15 millones de toneladas en 2018, siendo el mayor volumen de estas capturas provenientes de la industria pesquera, llevada a cabo por grandes embarcaciones industriales y de alta tecnología y orientada principalmente a la producción de harina y aceite de pescado (Flores, Bucaram, Paredes y Paredes, 2020). En la Figura I se muestra el aporte al Producto Bruto Interno (PBI) de la actividad pesquera industrial (Sociedad Nacional de Pesquería, 2018).

\section{Millones de Soles}

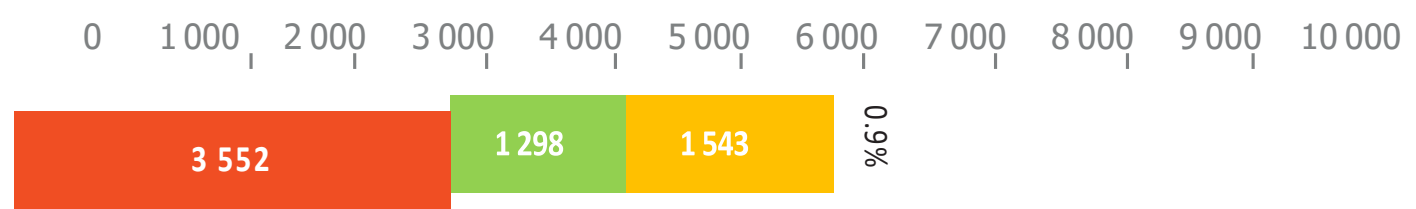

\begin{tabular}{|c|c|c|c|c|}
\hline 4980 & 1428 & & 2867 & $\underset{\ddot{\omega}}{\stackrel{⺊}{\circ}}$ \\
\hline 2017 & & & 2018 & \\
\hline Desembarques pesqueros & & 口 & Elaboración y preserv & pescado \\
\hline Elaboración de harina y aceite de pescado & & & Participación \% del PBI & \\
\hline
\end{tabular}

Fuente: Reporte de Sostenibilidad 2018, Sociedad Nacional de Pesquería

Gráfico I. Producto Bruto Interno de la actividad pesquera y participación en el PBI Nacional 2017 - 2018

En contraste, la pesca artesanal destina su producción principalmente al consumo humano directo para el mercado nacional, que a pesar de la diversidad de especies que se extraen los desembarques artesanales disminuyeron cerca del 35\% entre 2012 y 2017 a nivel nacional; en el caso del Puerto de Matarani en la Figura II se muestra su desempeño por volumen de desembarque (Aurum, 2018); mientras que el número de embarcaciones y pescadores artesanales incrementó entre $14 \%$ y 53\%, respectivamente a nivel nacional (Flores, D. et al., 2020). En la figura III, se presenta una distribución de las embarcaciones 
por especie extraída en la faena de pesca a nivel de la región de Arequipa (Ministerio de la Producción, 2012)

Fuente: Estudio de línea de base del sector pesquero artesanal del distrito de Islay, Aurum 2018

\section{Gráfico II. Evolución del volumen desembarcado en el puerto de Matarani 2006-2015 (TM)}

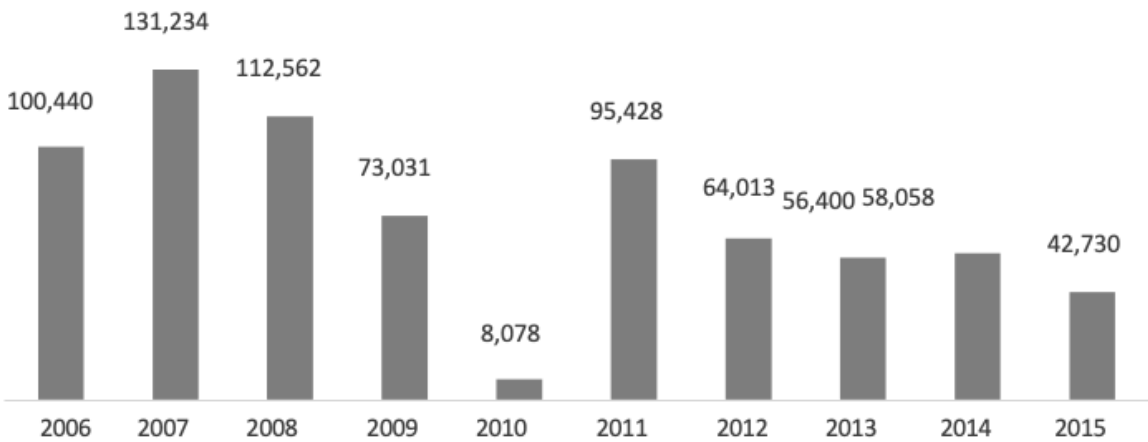

Fuente: I Censo Nacional de la Pesca Artesanal del ámbito marítimo, 2012. Produce

Gráfico III. Número de embarcaciones artesanales por especie extraída en las faenas de pesca. Arequipa vs. País

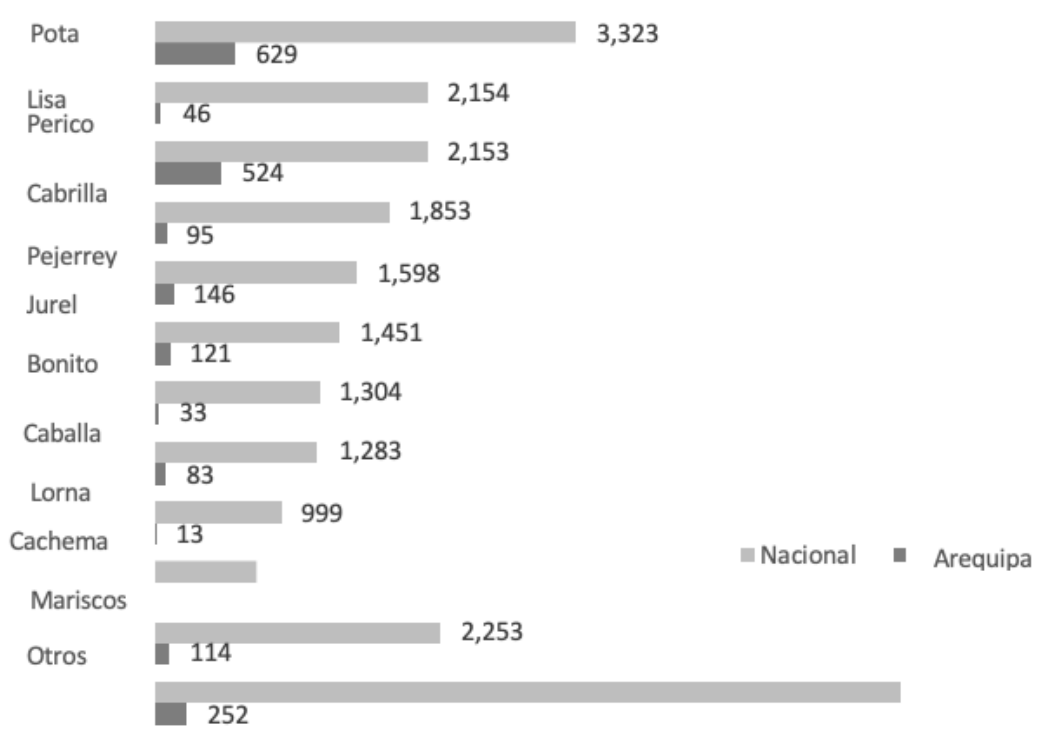

En este contexto, Arequipa es una de las regiones que tiene el litoral marino más largo del Perú y el Puerto de Matarani ha sido tradicionalmente una zona de importante 
actividad pesquera artesanal con diversidad de especies destinadas al consumo humano en el ámbito local y regional. Sin embargo, el impacto de la modernidad y la presencia de la pesca industrial (orientada principalmente a la extracción de anchoveta para la producción y exportación de harina de pescado), representan en la interacción un problema para el equilibrio del ecosistema marino y la vigencia de la pesca artesanal (Díaz, et al., 2016). A lo que se suma la ausencia de una política pública eficiente de ordenamiento pesquero que regule de manera integral el conjunto del sector, sin poner en riesgo la actividad artesanal, las especies y la seguridad alimentaria local. A su vez, la pesca artesanal es una actividad económica local que en su ciclo productivo absorbe cuantía mano de obra y dota de alimentos (recursos hidrobiológicos) a la localidad y la región por lo que es un elemento importante a considerar en el diseño de las estrategias de política pública del subsector.

Por otro lado, cabe señalar que los pescadores artesanales en su actividad pesquera cotidiana han desarrollado un importante conocimiento y practicas socioculturales sobre el mar, el comportamiento de las especies, los tiempos de pesca, etc. generando una conciencia de equilibrio ambiental la cual es importante identificar, revalorar e incorporar en las políticas públicas de desarrollo pesquero como elementos en las medidas de adaptación frente al cambio climático (Produce, 2020)

Bajo esta perspectiva, es importante que todos estos elementos mencionados se estudien y analicen no solo desde una vista económica-productiva sino que también se integren elementos culturales y antropológicos que revaloren al pescador artesanal y el imaginario sobre su actividad y su entorno, buscando desarrollar a partir de ello en contraste con una sólida estrategia de investigación científica algunos lineamientos de políticas públicas más integrales y no excluyentes, con el fin de generar un espacio de equilibrio y desarrollo integral del complejo pesquero en el distrito de Matarani. Por esta razón, en la presente investigación se aborda la pesca artesanal como un proceso que se configura a partir de una actividad productiva y un proceso cultural cuya relación a raíz del proceso de modernización establece un nuevo entorno sociocultural, ambiental y económico.

1. Consideraciones teóricas

1.1. Respecto a la pesca artesanal

Tratada la diversidad conceptual respecto a la definición de la pesca artesanal, puesto se han hecho alcances multidisciplinarios en relación al tema que nos ocupa, desde la biología, la antropología, la economía o el derecho; en el Cuadro 1 se presentan algunas de las más importantes aproximaciones teóricas. 


\section{Cuadro 1}

\section{Definiciones conceptuales de la pesca artesanal}

\begin{tabular}{|c|c|}
\hline$\overline{\mathrm{DR}}$ & IÓN \\
\hline $\begin{array}{l}\text { Ley General de la } \\
\text { Pesca (1992) }\end{array}$ & $\begin{array}{l}\text { Se considera actividad artesanal extractiva o procesadora, la } \\
\text { realizada por personas naturales, grupos familiares o empresas } \\
\text { artesanales, que utilicen embarcaciones artesanales o } \\
\text { instalaciones y técnicas simples, con predominio del trabajo } \\
\text { manual, siempre que el producto de su actividad se destine } \\
\text { preferentemente al consumo humano directo }\end{array}$ \\
\hline Alegret ( & $\begin{array}{l}\text { Pesca de bajura como un concepto genérico que incluye la pesca } \\
\text { artesanal, la de pequeña escala, la de litoral, la pequeña } \\
\text { producción mercantil pesquera, etc. }\end{array}$ \\
\hline McGodwil & $\begin{array}{l}\text { Hace uso del concepto de comunidades pesqueras en pequeña } \\
\text { escala, para referirse a aquellas que tienen como particularidades } \\
\text { principales la pequeña escala de sus inversiones de capital, } \\
\text { niveles de producción y poder político }\end{array}$ \\
\hline Neira (2005) & $\begin{array}{l}\text { La denominación como pescadores artesanales tiene también un } \\
\text { componente de auto identificación, es decir, de identidad }\end{array}$ \\
\hline Medicina ( & $\begin{array}{l}\text { Se define la pesca artesanal como una actividad pesquera que } \\
\text { utiliza las técnicas tradicionales con escaso desarrollo } \\
\text { tecnológico. }\end{array}$ \\
\hline $\begin{array}{l}\text { Guevara-Carrasco \& } \\
\text { Bertrand (2017) }\end{array}$ & $\begin{array}{l}\text { La pesca artesanal es una actividad extractiva realizada por } \\
\text { personas, grupos familiares o pequeñas empresas que a través del } \\
\text { tiempo operan las embarcaciones artesanales desarrollando } \\
\text { diferentes técnicas de pesca. }\end{array}$ \\
\hline
\end{tabular}

Fuente: Elaboración propia, 2021.

\subsection{Respecto al impacto de la modernidad}

El escenario sobre el que se circunscribe la pesca artesanal en el Puerto de Matarani es, a raíz del proceso globalizador, altamente complejo. Primero, porque los sistemas de extracción tradicional (pesca artesanal) se encuentran en permanente interacción con otros agentes económicos y sociales (industria pesquera, intermediarios, órganos de gobierno, organizaciones no gubernamentales, etc.). En segundo término, porque la dinámica de interrelaciones que se generan debido a estas lógicas de negociación y poder también admiten procesos de descomposición estructural, articulación, modernización y 
subordinación a los fines de expansión económica de los grandes capitales de inversión dentro de la industria pesquera (Saavedra, 2013).

En este sentido, es inevitable extrapolar del análisis la conjetura de que el sistema económico liberal sobre el que opera la industria de la pesca actúe como un mecanismo disolvente de los sistemas de producción y comercialización local, situación que se advierte empíricamente en la tensión de las organizaciones sociales de pescadores con las empresas industriales asentadas en el puerto, cuyo complejidad comunicacional reside fundamentalmente en la reducida capacidad local para articular respuestas económicas, ambientales y culturales sólidas frente al impacto de la modernidad, así cómo de establecer canales de comunicación fluidos entre los diferentes actores sociales en función de los factores externos.

En esta misma línea, integrando la figura de la industria pesquera al concepto de modernidad, el proceso de modernización se entiende como un sistema de cambios socioculturales, políticos, económicos, ambientales y tecnológicos que ocurren en una sociedad que se mueve hacia patrones más complejos y avanzados de organización, libertad, comunicación y democratización en todos los ámbitos pudiendo evidenciar su impacto en dos extremos de la realidad sociocultural: el primer impacto se da en el ámbito público y reside en un discurso consciente articulado que se cimenta desde arriba, principalmente por una pluralidad de instituciones que expresan sentimientos diversos y que no necesariamente se encuentran representados en las instituciones públicas que los representan. Por otro lado, el segundo impacto se canaliza en las formas privadas, las cuales en su mayoría están implícitas y con un grado de articulación hacia lo público mucho menor (pesca industrial).

Sin embargo, es innegable que el modelo de relaciones contrapuestas es insuficiente para analizar los escenarios presentes y futuros de la pesca artesanal, puesto que en la realidad se contrastan una gran pluralidad de expresiones pesquero-artesanales, que consecuentemente agudiza la complejidad de la interpretación de sus vinculaciones con el mercado, con las instituciones y con otros actores locales de su entorno (Saavedra, 2013).

\subsection{Respecto a las políticas públicas.}

Según el Gobierno Peruano, en la Guía de Políticas Nacionales del Centro Nacional de Planeamiento Estratégico (CEPLAN) y en el reglamento que regula las políticas nacionales en el país, se definen como políticas nacionales a "aquellas que constituyen decisiones de política a través de las cuales se prioriza un conjunto de objetivos y acciones 
para resolver un determinado problema público de alcance nacional y sectorial o multisectorial en un periodo de tiempo" (p.18).

Por otro lado, en esta misma línea las políticas públicas se pueden definir como el espacio principal de realización del pacto y/o contrato social entre Estado y sociedad, siendo un conjunto de actividades consensuadas entre la pluralidad de actores sociales que se canalizan directa o indirectamente a través de la estructura orgánica del Estado, y que se orientan a tener un impacto positivo sobre la vida y el bienestar de los ciudadanos. Es decir, son aquellas acciones de gobierno (mandato), que busca dar respuestas a las diversas demandas de la sociedad, entendiéndose esto como el uso estratégico de los recursos del tesoro público para cerrar las brechas de los problemas nacionales. En ese sentido, en la Figura I se muestra un resumen de los hitos normativos en relación a las regulaciones del sector pesquero artesanal en el Perú en contraste con el incremento de la flota pesquera artesanal.

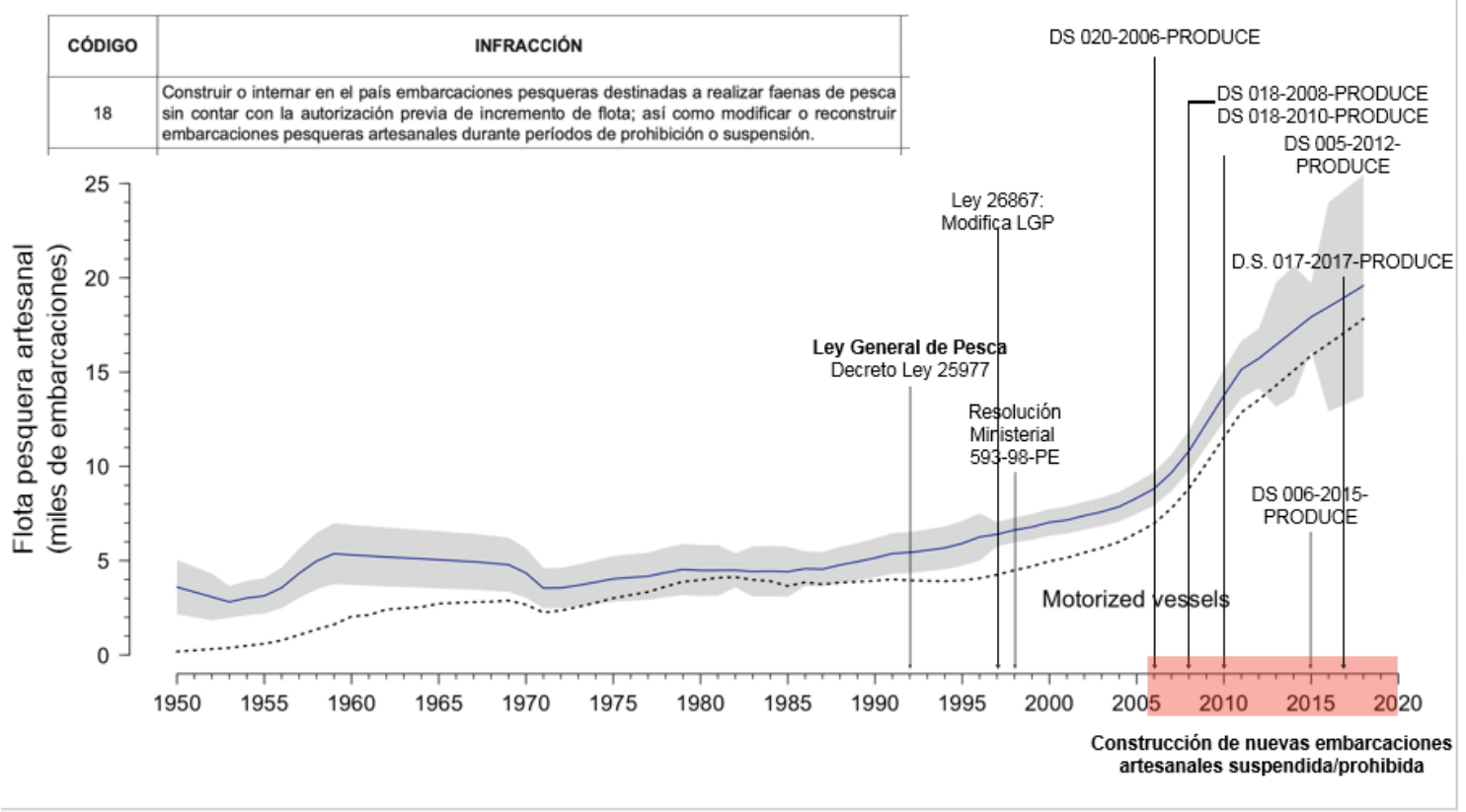

Fuente: Oceana Perú, 2020

\section{Figura I. Hitos normativos del sector pesquero artesanal en el Perú}

\section{Metodología}

Este estudio se ubica bajo el modelo cuantitativo, siendo una investigación correlacional, aplicando una metodología empírico-analítica bajo un modelo hipotético-deductivo. La técnica utilizada para el acceso a la información es la encuesta y entrevista como instrumentos. Se ha optado por un cuestionario estructurado y construido en función de diversas preguntas cerradas y de tipo narrativo. El universo de estudio estuvo constituido por los pescadores artesanales de la región de Arequipa que son aproximadamente 5,147 
pescadores artesanales según el I Censo Nacional de la Pesca Artesanal del Ámbito Marítimo 2012 y la población objeto de estudio han sido los pescadores artesanales del puerto de Matarani, que según el mismo censo ascienden a 1,500 pescadores artesanales. A través del muestreo probabilístico aleatorio simple con un nivel de confianza del 95\% $\mathrm{y}$ un error muestral del $\pm 5 \%$, la muestra final del estudio ha sido 306 pescadores artesanales. Para el análisis e interpretación de datos se utilizó el Statistical Package for the Social Sciences 20.0 (SPSS).

\section{Resultados de la investigación}

\subsection{Aspectos socio-económicos}

En cuanto al género de la población muestral de pescadores del Puerto de Matarani, los hombres representan el 88,56\% y las mujeres constituyen el $11.44 \%$ las cuales participan en su mayoría realizando actividades asociadas a la pesca propiamente dicha, una relación suplementaria que relega su participación a la labor realizada por su cónyuge, entre las más importantes la pesca de orilla o marisquería (recolección de algas y mariscos) que se ha ido perdiendo en favor del desarrollo de actividades comerciales.

En lo referido a la edad de los pescadores el grupo etario más representativo es el de 40 a 49 años de edad, $32.60 \%$ en el caso de los hombres y 4,25\% en el de las mujeres. Sin embargo, entre los 30 y 49 años se concentra más de la mitad de la muestra de estudio (hombres y mujeres) y por ende la mayor parte de la fuerza productiva de la actividad pesquera artesanal $(65,04 \%)$. Cabe resaltar en este punto, el rol de los viejos pescadores artesanales $(5,23 \%)$, aquellos hombres que fueron pescadores pero que por la edad y las limitaciones físicas dejaron la actividad y que son los custodios de los saberes ancestrales de la pesca artesanal.

Ahora bien, en cuanto al nivel profesional de los pescadores, la Tabla 1 evidencia la exigua formación superior de la población de pescadores en el puerto, siendo el $(9,14 \%)$ de la muestra de estudio el porcentaje de pescadores que han recibido formación superior (técnica y/o universitaria) y que en determinados casos no han concluido los estudios superiores de manera satisfactoria (técnica incompleta, universidad incompleta). Por otro lado, el mayor volumen de la población ha culminado sus estudios secundarios (46,73\%), y el $38,56 \%$ no los ha culminado satisfactoriamente, lo que denota la precariedad profesional del talento humano con casi nulos niveles de especialización $\mathrm{y}$ profesionalización, esto debido a los bajos ingresos que no permiten contar con capacidad de inversión en su crecimiento o realización personal (Medicina, 2014) 


\section{Tabla 1}

Grado de instrucción de los pescadores artesanales del Puerto de Matarani

\begin{tabular}{ccc} 
& $\mathrm{N}^{\circ}$ de Obs. & $\%$ \\
\hline Primaria incompleta & 1 & 0,33 \\
\hline Primaria completa & 8 & 2,61 \\
\hline Secundaria incompleta & 118 & 38,56 \\
\hline Secundaria Completa & 143 & 46,73 \\
\hline Técnica incompleta & 5 & 1,63 \\
\hline Técnica completa & 16 & 5,23 \\
\hline Universidad incompleta & 2 & 0,65 \\
\hline Universidad completa & 5 & 1,63 \\
\hline No sabe / no opina & 8 & 2,61 \\
\hline Total & 306 & 100.00
\end{tabular}

Fuente: Elaboración propia, 2021

\subsection{Problemática del sector pesquero artesanal}

Los problemas por los que atraviesa la pesca artesanal en el Puerto de Matarani, comprenden un complejo sistema de interacciones, de formas de comportamiento, de colectividades y de prácticas sobre su entorno y el medio marino que proyectan un escenario futuro difícil. En ese sentido, el problema de mayor preponderancia según la Tabla 2 es la precaria infraestructura de la cual disponen para ejercer su oficio, que comprende desde las estructuras portuarias (desembarque, almacenaje, cadena de frío) hasta la implementación de tecnología en sus embarcaciones debido a los obsoletos equipos en bienes de capital (Medicina, 2014). Otro de los factores críticos es la contaminación de la ecología marina derivada de las malas prácticas ejercidas en la extracción de los recursos hidrobiológicos, especialmente por los barcos industriales de arrastre que causan depredación y destrucción de los fondos marinos (Santos y Trimble, 2016). En tercer lugar, la informalidad en la que operan algunas embarcaciones de pescadores artesanales que no están asociados a las organizaciones sociales y que ejercen su labor de manera independiente y en muchos casos ilegal (Medicina, 2014) a la que cada día se adhieren más pescadores sin control alguno, lo que acarreará sin ninguna duda un costo muy alto para la sociedad. Por otro lado, otro factor de conflicto es la ineficiente presencia del Estado que a pesar de la implementación de programas de apoyo y fomento 
a través del Ministerio de la Producción no ha logrado fortalecer las capacidades de los pescadores.

Asimismo, uno de los problemas más apremiantes es la seguridad y salud del pescador, cuyo acceso a un seguro integral de salud como a un sistema de prestaciones sociales es limitado. Finalmente, cabe resaltar las contrariedades en los procesos de comercialización (precios bajos) comúnmente debido a la mediación de los intermediarios comerciales que juegan con los precios según sus intereses y el monopolio del mercado en los diferentes puertos, negociaciones que en su mayoría desfavorecen los ingresos y economía de los pescadores en relación al esfuerzo pesquero empleado.

Tabla 2

Principales problemas de la pesca artesanal en el Puerto de Matarani

\begin{tabular}{ccc} 
& $\mathrm{N}^{\circ}$ de Obs. & $\%$ \\
\hline Infraestructura inadecuada & 59 & 19.28 \\
\hline Contaminación ambiental & 43 & 14.05 \\
\hline Informalidad & 41 & 13.40 \\
\hline Salud del pescador & 35 & 11.44 \\
\hline Tecnología limitada & 32 & 10.46 \\
\hline Precios bajos & 28 & 9.15 \\
\hline Ineficiente gestión dirigencial & 24 & 7.84 \\
\hline Falta de apoyo del estado & 18 & 5.88 \\
\hline Malos equipos y materiales de trabajo & 17 & 5.56 \\
\hline Acceso de las embarcaciones & 12 & 3.92 \\
industriales & 12 & 3.92 \\
\hline Veda infringida & 11 & 3.59 \\
\hline Falta de agua & 6 & 1.95 \\
\hline Depredación de los recursos & 6 & 1.95 \\
\hline Desempleo & 21 & 6.85 \\
\hline Ninguno & 10 & 3.27 \\
\hline Otros & 23 & 549 \\
\hline
\end{tabular}

Fuente: Elaboración propia, varias respuestas por pescador, los resultados no suman 100\%, 2021 
Finalmente, a manera de reflexión, en el Cuadro 2 se presenta un análisis comparativo entre algunos factores a considerar en relación a los principales problemas de la pesca artesanal.

\section{Cuadro 2}

\section{Principales problemas de la pesca artesanal}

\begin{tabular}{|c|c|}
\hline FACTOR & PROBLEMA PRINCIPAL \\
\hline $\begin{array}{l}\text { Sostenibilidad de } \\
\text { recursos marinos. }\end{array}$ & $\begin{array}{l}\text { los Evidencia de disminución de capturas y tallas de especies de } \\
\text { interés comercial y de consumo local. }\end{array}$ \\
\hline $\begin{array}{l}\text { Planificación } \\
\text { ordenamiento. }\end{array}$ & $\begin{array}{l}\text { y Deficiencias en la política de ordenamiento del sector } \\
\text { pesquero. }\end{array}$ \\
\hline $\begin{array}{l}\text { Infraestructura } \\
\text { logística. }\end{array}$ & $\begin{array}{l}\text { y Altos costos de producción, precariedad de las estructuras } \\
\text { portuarias }\end{array}$ \\
\hline
\end{tabular}

Calidad e inocuidad. pesqueros en el mercado nacional e internacional

Consumo. Bajo consumo de los productos pesqueros nacionales.

\begin{tabular}{ll}
\hline Medidas & e \\
instrumentos de apoyo impacto de los programas de fomento en la gestión y \\
a la actividad. & manejo del sector pesquero artesanal.
\end{tabular}

Gobernanza Descentralización de la gestión y manejo participativo del
participativa. sector pesquero artesanal.

Formación $\quad \mathrm{y}$ Afiliación a sistemas de salud y de prestaciones sociales,
protección del recurso gestión del talento humano con bajos índices de humano. profesionalización y especialización

\begin{tabular}{ll}
\hline $\begin{array}{l}\text { Desarrollo } \\
\text { tecnológico }\end{array}$ & e Incipientes desarrollos tecnológicos e innovación en la \\
innovación. & actividad pesquera.
\end{tabular}

Seguimientos control Deficiencia y corrupción en los sistemas de seguimientos $\mathrm{y}$ vigilancia. control y vigilancia.

\begin{tabular}{ll}
\hline Gestión & y Agendamiento y gestión del cumplimiento de los acuerdos \\
cumplimiento & de internacionales.
\end{tabular}


acuerdos

internacionales.

Política y normativa.

Ausencia de una política nacional para el sector pesquero artesanal

Gestión de la Sistemas inestables y frágiles para la gestión de la información información. en el sector pesquero.

\begin{tabular}{ll}
\hline Mecanismos & de \\
Coordinación & Deficiencia en la coordinación y articulación institucional e \\
Interinstitucional & intersectorial. (Local, Regional y Nacional.)
\end{tabular}

Fuente: Elaboración propia a partir de (Carrillo, 2017)

\subsection{Hacia una política pública para la pesca artesanal: algunos lineamientos.}

El rol del Estado, como eje articulador, dentro del proceso de concepción de una política pública integral que permita mejorar la gestión del sector pesquero artesanal, es esencial. Con ese objeto se plantea que la política pública se enfoque en cinco componentes: cambio climático, gobernanza, fortalecimiento institucional, gestión de la información y gestión de la innovación e investigación.

\subsubsection{Lineamientos para la mitigación del cambio climático}

Se deben plantear los ejes de acción con el objeto de reducir la vulnerabilidad del sector pesquero artesanal frente al cambio climático, buscando impulsar la sostenibilidad ecológica del espacio haliéutico en relación a las factores abióticos y bióticos del ecosistema marino (Touré, Romagosa y Breton, 2015) recogiendo los lineamientos de adaptación planteados por el Ministerio de Producción (Produce, 2020) referidos a: el fortalecimiento de la pesca responsable; el fortalecimiento de la actividad acuícola para contribuir con la seguridad alimentaria, el pescado es una fuente de alimento económica y que constituye un importante suministro de nutrientes y proteínas (Guevara-Carrasco y Bertrand, 2017); la diversificación y fortalecimiento del valor agregado de la actividad pesquera; el fortalecimiento de los sistemas de modelado y predicción ante diferentes escenarios de cambio climático en el mar peruano y; la gestión del riesgo ecológico frente al cambio climático. Asimismo, es necesario identificar prácticas culturales en los mecanismos tradicionales del pescador, por ejemplo desde tiempos pre-incas se acostumbraba a encargar la administración de una determinada área marina a un grupo de pescadores para su extracción y resguardo, mecanismo que contribuye a garantizar la sostenibilidad de los recursos marinos en el área contribuyendo al fortalecimiento de la pesca responsable. 


\subsubsection{Lineamientos hacia una política de buena gobernanza (ordenamiento pesquero)}

Se plantean cuatro mecanismos de acción. En primer término la reformulación de fondos de apoyo y fomento de la pesca artesanal que deben estar orientados a promover el desarrollo sustentable del sector atendiendo los problemas de sostenibilidad que trae consigo la sobreexplotación de los recursos y apoyar los esfuerzos de las distintas organizaciones sociales de pescadores artesanales para mejorar sus condiciones sociales y laborales facilitando la transición del oficio del pescador a un negocio rentable dentro de los límites biológicos de seguridad.

En segundo término, el fomento de la asociatividad (formalización); los pescadores artesanales que pertenecen a alguna caleta están afiliados en primer grado a sindicatos, asociaciones gremiales (Ospas), siendo necesario promover la asociatividad como mecanismo de ordenamiento a través de diferentes estrategias como la generación de entidades jurídicas para mejorar la explotación de las pesquerías, producción y comercialización de toda la producción de sus miembros (Gonzáles et al., 2013), este planteamiento tipifica a la caleta como un órgano productivo funcional cuyo reto está en la generación de climas de negocio idóneos y un sistema efectivo de extracción, manejo y venta de los productos.

Como tercer mecanismo, el fomento de la inversión público privada en infraestructura portuaria; que en el caso de Matarani deviene en la construcción del nuevo desembarcadero, pero cuya política de infraestructura debiera tener una estrategia clara en relación a las inversiones progresivas en el tiempo, orientadas más allá del equipamiento de las embarcaciones sino a cuestiones de transformación y comercialización que le otorgan un valor agregado a las capturas, dotando de estructuras portuarias mínimas a las distintas caletas anulando de esta manera los desequilibrios en relación a los grandes puertos del país y del mundo en comparación con la actividad industrial.

Cuarto, la implementación de un sistema de cogestión y gobernanza para la coordinación entre la pluralidad de actores involucrados, que permita la redefinición de las reglas que organizan la participación de los actores y la gestión de los recursos, así como la inclusión de criterios éticos y políticos en la gestión técnica y la retroalimentación de conocimientos y saberes (científicos, técnicos y tradicionales) abordando la gestión pesquera desde una perspectiva de sostenibilidad (Florido, 2013) 
Por otro lado, cabe agregar que el ordenamiento pesquero es imprescindible para lograr la sostenibilidad de la pesca artesanal así como para la protección de los medios de vida de las comunidades que dependen de la pesca. En relación al enfoque sobre el crecimiento azul (FAO, 2016) la promoción de medios de vida sostenibles para las comunidades pesqueras costeras, la protección social y las condiciones de trabajo decente a lo largo de la cadena de valor del pescado son elementos estructurantes en su implementación.

Finalmente, en relación a lo presentado anteriormente la propuesta radica en concebir la infraestructura pesquera artesanal como un centro de articulación entre la actividad extractiva y el mercado (Galarza y Kámiche, 2015) como se describe en el modelo presentado en la Figura II.

4.

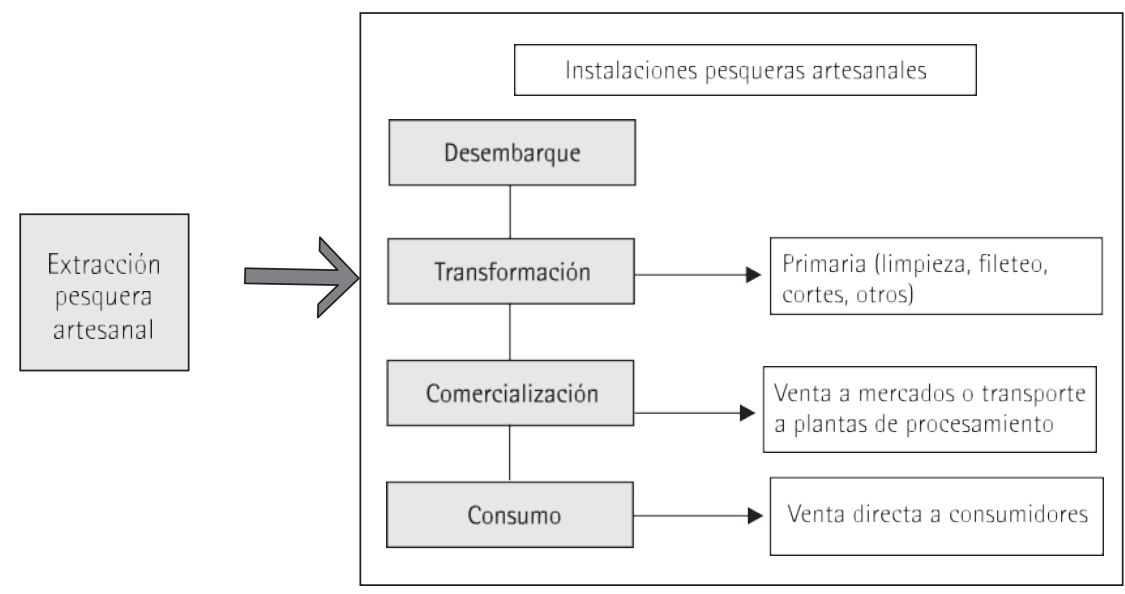

Fuente: Elaboración propia a partir de (Galarza y Kámiche, 2015)

Figura II. Propuesta de cadena de valor de la pesca artesanal

\subsubsection{Lineamientos para el fortalecimiento institucional}

Se debe constituir una Política Nacional de Pesca que favorezca el diseño de los objetivos de la gestión de la pesca artesanal a largo plazo (Flores et al., 2020) cuyo énfasis en materia de fortalecimiento institucional se sitúe en las siguientes cinco líneas de acción. Primero, la regulación del sistema de desembarque y descarga; mediante el control de las operaciones en las transacciones comerciales, para lo cual es necesario delimitar los lugares de desembarque y descarga, Segundo, la regulación de los intermediarios comerciales; en el muelle del puerto, los pescadores ofrecen la producción mediante el sistema de subasta a los mayoristas y minoristas (tipo holandés o a la baja), pero el comportamiento de los precios, su variabilidad y oscilación no obedece necesariamente a 
la dinámica de la oferta y la demanda o a la calidad del producto sino a otras causas relacionadas con las estrategias especuladoras de los intermediarios (González et al., 2013) siendo necesario generar canales de venta directa dirigidos al cliente final dando soporte al pescador en un mercado virtual y con fuerza comercial propia, promoviendo la desintermediación y abriendo el mercado de los productos de la pesca artesanal a nivel nacional (generación de valor agregado en los productos) y estableciendo un equilibrio entre el esfuerzo pesquero y los ingresos del pescador.

Tercero, el mejoramiento de los sistemas de comercialización de los productos; los canales de comercialización en el puerto de Matarani mantienen una relación estrecha con intermediarios y plantas, en ese sentido la propuesta pasa por la necesidad de incrementar y redirigir los esfuerzos a los proyectos de mejora y transformación de los productos derivados de las faenas de pesca, mejorando las condiciones sanitarias del recurso hidrobiológico y aplicando procesos simples con nuevas tecnologías que desarrollen métodos innovadores de producción, comercialización y venta de productos procedentes de los desembarques locales (por ejemplo una planta procesadora que le dé valor agregado al producto: pescado ahumado, fileteado, deshidratado, merchandising, etiquetado) entro otras estrategias que además de integrar el rol de la mujer en el proceso de transformación conducen a "pescar menos y ganar más".

Cuarto, la descentralización del modelo institucional pesquero; puesto que es evidente el distanciamiento entre el pescador artesanal y los diferentes organismos gubernamentales, que en torno al desarrollo sostenible de las zonas de pesca, la política de descentralización debiera proporcionar a las comunidades de pescadores las herramientas necesarias para adoptar una estrategia de desarrollo integral en sus territorios, reconociendo su condición sociocultural y las necesidades locales en relación al proceso de modernización del aparato productivo (Marin, 2007).

Finalmente, se debe estimular el desarrollo del capital humano como elemento transversal en el proceso de desarrollo local a través de programas de educación, enseñanza y extensión (Pazmiño, Gallardo, Ortiz y Beltrán, 2017) que permita elevar el nivel de formación y profesionalización de los pescadores artesanales.

\subsubsection{Lineamientos para la gestión de la información}

La sobreexplotación de los recursos marinos está dando lugar a la reducción de la captura de la flota pesquera artesanal en las comunidades de pescadores (Ramírez, 2015). En materia de gestión de la información se plantean tres líneas de acción. La primera se refiere a la implementación de un sistema que recopile y sistematice las bases de datos de 
todas las instituciones involucradas en el proceso de la pesca artesanal, estandarizando las metodologías de generación y actualización de información, para afianzar la toma de decisiones y mejorar las medidas de manejo actuales (Flores et al., 2020). En segundo lugar, es necesaria la ejecución de un nuevo censo de la pesca artesanal que permita disponer de información actualizada y relevante para la gestión de la pesca artesanal.

Tercero, en este punto es cardinal la lucha contra la eliminación de la pesca ilegal que pone en riesgo la sostenibilidad de los recursos hidrobiológicos y la seguridad alimentaria de las poblaciones que dependen de estos recursos. En este marco, el sistema de control de las embarcaciones de la pesca artesanal debe garantizar el registro integral y la prohibición de circulación en medio marítimo así como el ejercicio de actividades de pesca a todas las embarcaciones que no estén registradas y no dispongan de un dispositivo que permita su detección e identificación mediante sistemas de control remoto (González et al., 2013). Asimismo, se debe facilitar la asistencia técnica necesaria para robustecer los sistemas de vigilancia, monitoreo y control de la pesca contribuyendo a la conservación de los ecosistemas marinos a través de una adecuada gobernanza (Comisión de Pesca Continental y Acuicultura para América Latina y El Caribe (COPESCAALC), 2018)

\subsubsection{Lineamientos para la gestión de la innovación e investigación}

En términos de innovación, es necesario aplicar nuevos enfoques como el stakeholder approach, la gobernanza interactiva o la ecología de saberes (Florido, 2013) para ubicar las perspectivas de la pesca artesanal en una dinámica diferente. Por ejemplo, en relación a la patrimonialización de la cultura pesquera, se debe configurar una propuesta de valoración con diversos componentes (gastronomía, turismo en zonas portuarias, reconocimiento de las pesquerías tradicionales en el entorno local), que complemente la labor estrictamente ligada al acto extractivo a través de experiencias no convencionales, que amplían los elementos culturales dotados de alto valor identitario (la gastronomía por ejemplo) en función de estrategias comerciales como el turismo vivencial.

Por otro lado, en materia de investigación, según la Tabla 3 se presentan algunas de las necesidades de investigación reconocidas por los pescadores artesanales del Puerto de Matarani.

Tabla 2

Estudios, proyectos e investigaciones a desarrollar para la pesca artesanal en el Puerto de Matarani 


\begin{tabular}{|c|c|c|}
\hline & $\begin{array}{l}\mathrm{N}^{\circ} \text { de } \\
\text { Obs. }\end{array}$ & $\%$ \\
\hline Desarrollo de $\mathrm{I}+\mathrm{D}+\mathrm{i}$ & 214 & $69,93 \%$ \\
\hline $\begin{array}{c}\text { Desarrollo tecnológico (equipamiento, } \\
\text { instrumentos) }\end{array}$ & 12 & $3,92 \%$ \\
\hline Impacto ambiental (recursos, océano) & 11 & $3,59 \%$ \\
\hline Parque industrial & 9 & $2,94 \%$ \\
\hline $\begin{array}{l}\text { Búsqueda de nuevos mercados } \\
\text { (comercialización) }\end{array}$ & 8 & $2,61 \%$ \\
\hline Benchmarking (productos) & 6 & $1,96 \%$ \\
\hline Seguridad marina & 4 & $1,31 \%$ \\
\hline Áreas de repoblamiento y bancos naturales & 3 & $0,98 \%$ \\
\hline Sistemas de congelamiento de los productos & 1 & $0,31 \%$ \\
\hline No sabe / no responde & 78 & $25,49 \%$ \\
\hline TOTAL & 306 & $100 \%$ \\
\hline
\end{tabular}

Fuente: Elaboración propia, varias respuestas por pescador, los resultados no suman $100 \%, 2021$

\section{Conclusiones}

La falta de articulación entre el Estado, la industria y las comunidades de pescadores artesanales ha trabado el crecimiento y dinámica de la pesca artesanal, posibilitando el desarrollo de la informalidad y desorden en la gobernanza del sector (ordenamiento pesquero). Por otro lado, en el nuevo contexto de la gestión de la pesca artesanal caracterizada por el impacto de la modernidad, queda claro que su análisis no puede centrarse en enfoques fragmentados sino en procesos estrictamente multidisciplinarios para evitar estudios o políticas sesgadas.

Además, existen procesos que habiendo sido particulares de la industria pesquera, se han incorporado a la práctica artesanal como son el uso de nuevas tecnologías, la intensificación de la producción y la comercialización de carácter cosmopolita, lo cual obliga a repensar el concepto de pesca artesanal pues no podemos asumir que los pescadores artesanales conserven su dinámica tradicional y de equilibrio ecológico, en un contexto de alta competitividad comercial, intensiva tecnologización y reconfiguración de las relaciones laborales. 
Finalmente, se concluye que la propuesta de política pública de la pesca artesanal en el Perú, en el caso específico de Islay Matarani, debe poner de manifiesto todos los aspectos que aquejan al sector y que se recogen en la presente investigación (cambio climático, ordenamiento pesquero, fortalecimiento institucional, gestión de la información y gestión de la innovación), formulando estrategias y objetivos para el desarrollo del sector. Si bien cada uno de los objetivos constituye un reto importante, en muchos casos son ineludibles si se quiere llegar a la sostenibilidad de la pesca artesanal más aún si se pretende conseguir una buena gobernanza de los recursos del mar, no solo en el puerto de Matarani sino en cada uno de los puertos del país.

\section{Referencias bibliográficas}

Alegret, J. (2002). Gobernabilidad, legitimidad y discruso cientifico: el papel de las ciencias sociales en la gestión de la pesca de bajura. Esuskalerria: Zainak. Cuadernos de Antropología-Etnografía.

Aurum. (2018). Estudio de línea de base del sector pesquero artesanal del distrito de Islay. Islay Matarani: Sociedad Minera Cerro Verde.

Carrillo, V. (2017). Análisis del agendamiento de la política pesquiera en Colombia ¡del pez grande al pez pequeño? Tesis de Maestría. Bogotá: Universidad Externado de Colombia.

CEPLAN. (2018). Guía de Políticas Nacionales. Lima: CEPLAN.

Comisión de Pesca Continental y Acuicultura para América Latina y El Caribe (COPESCAALC). (2018). Informe de avance en la aplicación de las conclusiones y recomendaciones de la Décimo cuarta reunión ordinaria de la comisión. Ciudad de Panamá: FAO. Obtenido de http://www.fao.org/3/I8416ES/i8416es.pdf

De la Puente, S., López, R., Benavente, S., Sueiro, J., \& Pauly, D. (2020). Growing into poverty: reconstructing peruvian small-scale fishing effort between 1950 and 2018. Frontiers in Marine Science, 7(681). doi:10.3389/fmars.2020.00681

Díaz, J., Guillot, L., \& Velandia, M. (2016). La pesca artesanal en la costa norte del Pacífico colombiano:un horizonte ambivalente. Bogotá, Colombia: Fundación MarViva. 
Flores, D., Bucaram, S., Paredes, E., \& Paredes, E. (09 de Diciembre de 2020). Hablemos de sostenibilidad y cambio climático. (B. I. Desarrollo, Editor) Recuperado el 03 de Febrero de 2021, de https://blogs.iadb.org/sostenibilidad/es/propuesta-para-eldesarrollo-de-la-pesca-artesanal-en-peru/

Florido, D. (2013). Encrucijadas para los sociedades pesqueras del Sur en el marco de la globalización. Líneas de investigación y perspectivas teórico-metodológicas. Revista Andaluza de Antropología(04), 1-9.

Galarza, E., \& Kámiche, J. (2015). Pesca artesanal: oportunidades para el desarrollo regional. Lima: Universidad del Pacífico.

Gobierno del Perú. (21 de Diciembre de 1992). Ley General de la Pesca. Lima, Perú: El Peruano.

González, E., Cerda, R., Quezada, J., Martinez, G., López, E., Thomas, F., \& Merino, J. (2013). Propuesta de Política Pública de Desarrollo Productivo para la Pesca Artesanal. Chile: AGCI. Programa de Innovación y Competitividad.

Guevara-Carrasco, R., \& Bertrand, A. (2017). Atlas de la pesca artesanal del mar del Perú. Lima, Perú: IMARPE-IRD.

Marin, W. (2007). Cultura y modernización de la pesca artesanal en Chile: Adaptaciones, cambios e hibridaciones en una caleta de algueros. $\operatorname{Mad}(17), 113-143$.

McGoodwin, J. (2002). Comprender las culturas de las comunidades pesqueras, clave para la ordenación pesquera y la seguridad alimentaria. Roma: FAO.

Medicina, J. (2014). Pesca Artesanal en el Perú. Ingeniería Industrial(032), 27-58. doi: https://doi.org/10.26439/ing.ind2014.n032.115

Ministerio de la Producción. (2012). I Censo Nacional de la Pesca Artesanal del Ámbito Marítimo. Perú: Produce.

Neira, P. (2005). Las comunidades de pescadores artesanales frente a la modernización: el caso de la Caleta Queule. Tesis para obtar el título de Antropología Social. Santiago: Universidad de Chile. 
Organización de las Naciones Unidas para la Alimentación y la Agricultura (FAO). (2016). La Agenda 2030, los objetivos de desarrollo sotenible y la pesca y acuicultura. Roma: FAO. Obtenido de http://www.fao.org/3/a-mq652s.pdf

Organización de las Naciones Unidas para la alimentación y la Agricultura. (2019). Estadísticas de pesca y acuicultura 2017. Roma: ONU.

Pazmiño, G., Gallardo, W., Ortiz, S., \& Beltrán, D. (2017). La implementación de políticas públicas como el mecanismo para el desarrollo del talento humano y de los sectores pesqueros del Ecuador. Comercio y Negocios(7), 84-107.

Produce. (2020). Diagnóstico del sector pesquero y acuícola frente al cambio climático y lineamiento de adaptación. Lima: Ministerio de la Producción.

Ramírez, J. (2015). Evidenciando la necesidad de gestión en la pesca artesanal de Colombia. El caso de la pesca artesanal Wayuu en La Guajira, Caribe colombiano. Acuicultura y pesca con responsabilidad, 1, 78-82.

Saavedra, G. (2013). La pesca artesanal en las encrucijadas de la modernización. Usos, apropiaciones y conflictos en el borde costero del Sur de Chile. Revista Andaluza de Antropología(04), 79-102.

Santos, P., \& Trimble, M. (Mayo-junio de 2016). Del desarrollo al bienestar, los consensos culturales nunca son finales: aprendizajes de los pescadores artesanales de Piriápolis, Uruguay. Estilos de vida, interacciones sociales y desarrollo humano. Conferencia llevada a cabo en la VI Conferencia de Alcadeca. Montevideo.

Sociedad Nacional de Pesquería. (2018). Reporte de Sostenibilidad 2018. Reporte GRI del sector de pesca industrial para ingredientes marinos. Lima: SNP.

Touré, E., Romagosa, F., \& Breton, F. (2015). Análisis de la situación de la pesca artesanal en el delta y valle bajo del río Senegal a partir del marco conceptual DPSIR. Estudios Geográficos, $\quad$ LXXVI(279), $\quad$ 703-731. doi:10.3989/estgeogr.201525 